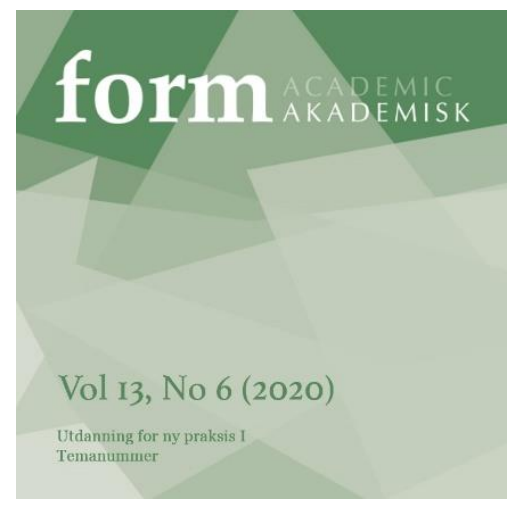

\title{
Virtual Reality in Design Processes
}

\section{- a literature review of benefits, challenges, and potentials}

\begin{abstract}
Virtual Reality (VR) opens new possibilities in the fields of architecture, design and engineering. If combined with Building Information Modelling (BIM) or simpler 3D models, it could be possible to walk into buildings not yet built or to examine designed objects in three dimensions before they are made. This literature review examines studies in which VR was used in architecture, design and engineering as part of design processes. The review highlights promising benefits, such as increased understanding of complex issues concerning design tasks, size and dimensions. At the same time, several challenges are revealed, such as the inability of VR-systems to offer satisfactory functionalities for sketching and designing. Finally, the author discusses how VR can be implemented in relevant subjects in lower and upper secondary school.
\end{abstract}

Keywords:

Virtual Reality (VR), Architecture, Design, Education, Art and crafts

\section{INTRODUCTION}

This article reviews the use of Virtual Reality (VR) in the fields of architecture, design and engineering as part of the design process across 15 international research studies published in the 2015-2020 period. The results of the review are used to discuss how VR can be implemented in art and design in lower and upper secondary education. To provide additional information on extant research in this area, four previous literature reviews on the use of VR in education are presented. Before doing so, however, the concept of VR must be introduced.

VR places users in a fully virtual environment in which all information is decorrelated from the users' real-world space (Parveau \& Adda, 2018). VR can be classified into three categories: immersive, semi-immersive and non-immersive (Baus \& Bouchard, 2014; Ma \& Zheng, 2011; Maas \& Hughes, 2020; Zahabi \& Abdul Razak, 2020). In an immersive VR system, the user wears a Head Mounted Display (HMD), completely filling the user's field of view, or is placed in a room with large screens on three or four walls, ceiling and/or on the floor, such as a Cave Automatic Virtual Environments (CAVE) or an immersive virtual environment (IVE). In addition to visual input, auditory and tactile sensory aspects may be delivered. The user can control the system with the use of, e.g. a joystick, hand-held sensors, gloves or bodysuits. A non-immersive VR system uses a conventional graphics workstation with a 
monitor, keyboard and mouse, with a virtual environment displayed on the monitor. Semi-immersive systems are similar to non-immersive systems but use larger screens or high-resolution projections, giving the user a wider field of view and a higher degree of realism.

Levels of immersion can be objectively assessed based upon parameters such as field of view, frame rate, headtracking or supported haptics, as demonstrated in this three category-classification of VR. The feeling of being present in a virtual environment is a reaction to immersion, meaning that presence and immersion are related, but different people may report different levels of presence to the same immersive system (Slater et al., 2009). Experimental studies suggest that the way data are displayed and how the user is able to interact with the system are more important than the level of realism, as the user may fill in absent sensory information by cortical processing (Sanchez-Vives \& Slater, 2005; Slater et al., 2009). The embodiment of technology is another interesting aspect of the classification of VR. Technology worn by the users, such as HMDs, may be perceived as part of their bodies and thus essentially 'disappear' (Tussyadiah et al., 2017). In contrast, non-immersive or semiimmersive VR technology is detached from the users' bodies.

VR is often placed on a Reality-Virtuality continuum, as defined by Milgram and Kishino (1994). On this continuum, virtual environments are placed in opposition to real environments, which include only real objects. Between these poles is Mixed Reality (MR), which consists of hybrid displays with different levels of Reality and Virtuality (Milgram \& Kishino, 1994). Building on Milgram and Kishino's Reality-Virtuality continuum, Juraschek et al. (2018) developed a model that shows how different technology applications are placed within the continuum (Figure 1). Semi-immersive VR is placed relatively close to the middle of the continuum, as the user is simultaneously aware of both the real and virtual worlds, whereas 'in immersive VR, the user is experiencing an almost total virtual environment' (Juraschek et al., 2018, p. 154). At the middle and towards the real environments, the model place concepts of Augmented Reality (AR). In AR, virtual elements are overlaid on the real world, e.g. through wearable holographic devices, such as HoloLens, or through the screens of mobile phones, most famously in the gaming app Pokémon Go. Although this article focuses solely on VR, AR is often included in research articles on VR, as it is a related technology, and will therefore be mentioned when relevant.

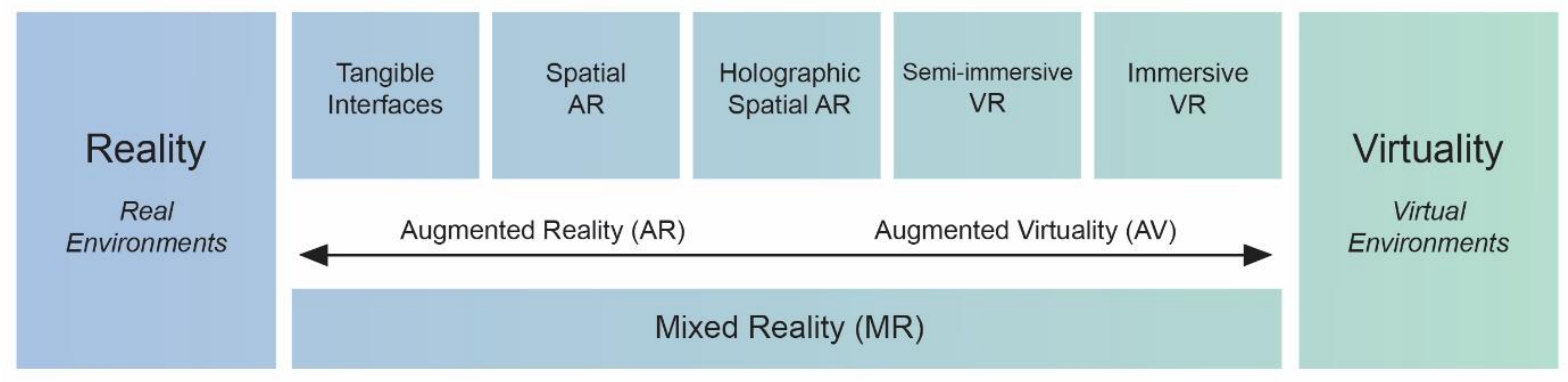

FIGURE 1. Reality-virtuality continuum based on Milgram and Kishino (1994), with the allocation of technology applications by Juraschek et al. (2018).

HMD systems are generally quite expensive, even though prices vary, so it is unlikely that public schools will be able to afford them in the near future. A simpler and significantly cheaper alternative is Mobile $V R$, which places smartphones in VR glasses made from cardboard and plastic lenses. Free or inexpensive apps allow the user to become immersed in 3D models or other virtual environments. If the feeling of being present is not dependent on highly detailed, realistic graphics (Sanchez-Vives \& Slater, 2005; Slater et al., 2009), then cheaper systems like this may be a sufficient alternative. With the concept of VR having been introduced, four literature reviews on the use of VR in education can now be presented.

The potentialities of VR can only be reached with immersive VR, according to Freina and Ott (2015). In their literature review of articles published in 2013-2014, they looked specifically at advantages and potentials in the use of immersive VR in education. They found that the main motivation 
for using VR is to have otherwise inaccessible experiences, such as visiting historical periods or outer space, dangerous experiences, such as firefighting, or unethical experiences, such as surgery training. Adding gaming elements to the experiences increases the learners' involvement and motivation. Freina and Ott also found that most of the articles they reviewed concerned VR in higher education and adult training, implying that little research had been done on VR in education in primary and secondary school.

In their systematic review of 99 papers published between 2010 and June 2017, Kavanagh et al. (2017) focused specifically on motivations behind and problems with the educational application of VR. Their analysis showed that VR is used frequently only in situations where realistic simulations are needed or for training purposes. The most prevalent motivation found was 'the belief that students would be motivated by the novelty of VR technologies; a factor which would likely diminish with continual use' (Kavanagh et al., 2017, p. 109). Reported problems with the use of VR were most commonly linked to the cost of equipment and the training of users, as well as software and hardware usability, particularly the occurrence of software usability issues (Kavanagh et al., 2017).

Aguayo et al. (2017) conducted a literature review with the aim of capturing the range and scope of key themes in mobile learning from 2010 to 2016, with special attention to Mobile AR and VR. A relatively low uptake of mobile learning, especially the use of learner-generated content and contexts, was found. According to Aguayo and colleagues, mobile learning has not yet lived up to its full potential, as teachers often apply the new technology to their old educational practices. Consequently, the authors argue for the implementation of learner-centred practices that focus more on the learning process, moving away from rigid structures and apps as content-delivery platforms (Aguayo et al., 2017).

Merchant et al. (2014) examined the learning outcomes of desktop VR-based instructions in their meta-analysis of 67 articles published up until November 2011. They found that the use of games, simulations and virtual worlds had an overall positive effect on students' learning. Most effective was the use of game-based learning, although here the analysis also revealed evidence of the novelty effect, as the quality of learning outcomes diminished as students spent more time playing the games. Both games and virtual worlds led to higher achievement levels on knowledge-based, ability-based and skillbased measures, but the simulations failed to improve skill-based learning outcomes. As this may be attributable to students spending less time working with simulations, increasing the amount of time they spend on simulations may also yield positive effects on their skills acquisition (Merchant et al., 2014).

Based upon these reviews, it is clear that VR is often used for realistic simulations or skill-training purposes, but that it is important to spend enough time on the simulations in order to improve skills. Some argue that VR and mobile learning have not yet lived up to their potential and that many teachers continue to follow their old educational practices even when adding new technology, sometimes with the belief that the novelty of the technology will be sufficient to motivate the students. Aguayo et al. (2017) called for greater focus on learner-centred practices instead of using platforms that deliver premade content. Much of the research included in these reviews was conducted in the area of higher education, and there is therefore a need for more research on VR in primary and secondary school.

The aim of this article is twofold. First, it investigates how the selected articles describe the use of VR in design processes in the fields of architecture, design and engineering. Second, it seeks to identify the reported benefits and challenges of VR use. The two research questions guiding this review are as follows:

1. How is the use of virtual reality (VR) as part of design processes described in the selected articles?

2. Which benefits and challenges are reported from the use of VR in design processes?

In the next section, the literature search method, article selection criteria and analytical approach are described. This is followed by a presentation of the findings with regard to the two research questions. Building upon these findings, the transferability of VR to Art and crafts in lower secondary school, as well as to related subjects in upper secondary school, is discussed. Lastly, the article concludes with some final remarks. 


\section{METHODS}

This article constitutes a narrative review, as derived from Greenhalgh et al. (2018). This approach allows for the incorporation of different knowledge sources and reviewing literature that employs different methods. The results are of a qualitative nature, aiming at a deeper understanding through interpretation and critique (Greenhalgh et al., 2018). The literature search and article selection were conducted systematically. The procedures used are described here in detail to provide the basis for future replication of the searches and to avoid bias. The initial literature search was conducted in February 2020 using the following databases: Academic Search Ultimate, ERIC, Education Source and Engineering Village. In the database searches, the search strings shown in Table 1 were used. As VR is a technology undergoing rapid development, a short time frame was chosen for the literature search. After both an automatic and manual removal of duplicates, the search yielded 1017 results.

TABLE 1. The two separate searches in four databases conducted in February 2020 with their search strings. The number of results includes duplicates.

\begin{tabular}{|c|c|c|c|}
\hline $\begin{array}{l}\text { NAME OF } \\
\text { DATABASE }\end{array}$ & SEARCH STRING & LIMITER/EXPANDER & $\begin{array}{l}\text { NUMBER OF } \\
\text { RESULTS }\end{array}$ \\
\hline $\begin{array}{l}\text { Academic } \\
\text { Search } \\
\text { Ultimate, } \\
\text { ERIC, } \\
\text { Education } \\
\text { Source }\end{array}$ & $\begin{array}{l}\text { (“Design" OR "art* and craft*" OR "visual art*” OR } \\
\text { "architecture" OR "BIM") AND (“VR" OR “Virtual reality") } \\
\text { AND (“Education" OR "student" OR "design literacy") }\end{array}$ & $\begin{array}{l}\text { Limiters - Peer Reviewed; } \\
\text { Date Published: } \\
\text { 20150101-20201231 } \\
\text { Narrow by Language: - } \\
\text { english } \\
\text { Search modes - } \\
\text { Boolean/Phrase }\end{array}$ & 831 \\
\hline $\begin{array}{l}\text { Engineering } \\
\text { Village }\end{array}$ & $\begin{array}{l}\text { (((((("Design" OR "art* and craft*" OR "visual art*" OR } \\
\text { "architecture" OR "BIM") WN ALL) AND (("VR" OR "Virtual } \\
\text { reality") WN ALL)) AND (("Education" OR "student" OR } \\
\text { "design literacy") WN ALL))) AND (((ja\} WN DT) AND } \\
\text { (\{english\} WN LA) AND ((2020 OR } 2019 \text { OR } 2018 \text { OR } 2017 \\
\text { OR } 2016 \text { OR 2015) WN YR))) }\end{array}$ & & 604 \\
\hline
\end{tabular}

In order to include more articles, additional searches were conducted in November 2020. The main addition was the Scopus database, as well as supplementary searches in Academic Search Ultimate, ERIC, Education Source and Engineering Village aimed at including articles published after the previous search. In the database searches, the search strings shown in Table 2 were used. The search yielded 1089 results after both an automatic and manual removal of duplicates.

The process of selecting articles involved several rounds of inclusion and exclusion based on reading titles, reading abstracts, gathering full texts and reading full articles. This process followed the inclusion and exclusion criteria shown in Table 3. The article selection process is also visualised in a flow chart, shown in Figure 2. 
TABLE 2. The three separate searches in five databases conducted in November 2020 with their search strings. The number of results includes duplicates.

\begin{tabular}{|c|c|c|c|}
\hline $\begin{array}{l}\text { NAME OF } \\
\text { DATABASE }\end{array}$ & SEARCH STRING & LIMITER/EXPANDER & $\begin{array}{l}\text { NUMBER OF } \\
\text { RESULTS }\end{array}$ \\
\hline Scopus & $\begin{array}{l}\text { ( ( "Design" OR "art* and craft*" OR "visual art*" OR } \\
\text { "architecture" OR "BIM" OR "Building Information Modeling" } \\
\text { ) own AND all AND ( ( "var" OR "Virtual reality" ) own AND } \\
\text { all ) ) AND ( ( "Education" OR "student" OR "design literacy" } \\
\text { ) ) AND ( LIMIT-TO ( PUBYEAR, 2021) OR LIMIT-TO ( } \\
\text { PUBYEAR, 2020 ) OR LIMIT-TO ( PUBYEAR, 2019) ) OR } \\
\text { LIMIT-TO ( PUBYEAR, 2018 ) OR LIMIT-TO ( PUBYEAR, } 2017 \\
\text { ) OR LIMIT-TO ( PUBYEAR, 2016) ) OR LIMIT-TO ( PUBYEAR, } \\
\text { 2015 ) ) AND ( LIMIT-TO ( SUBJAREA, "COMP" ) OR LIMIT-TO } \\
\text { ( SUBJAREA, "SOCI" ) OR LIMIT-TO ( SUBJAREA, "ENGI" ) OR } \\
\text { LIMIT-TO ( SUBJAREA, "ARTS" ) ) AND ( LIMIT-TO ( PUBSTAGE } \\
\text {, "final" ) ) AND ( LIMIT-TO ( DOCTYPE, "ar" ) OR LIMIT-TO ( } \\
\text { DOCTYPE, "cP" ) OR LIMIT-TO ( DOCTYPE, "re" ) ) AND ( } \\
\text { LIMIT-TO ( LANGUAGE, "English" )) }\end{array}$ & & 879 \\
\hline $\begin{array}{l}\text { Academic } \\
\text { Search } \\
\text { Ultimate, } \\
\text { ERIC, } \\
\text { Education } \\
\text { Source }\end{array}$ & $\begin{array}{l}\text { ("Design" OR "art* and craft*" OR "visual art*" OR } \\
\text { "architecture" OR "BIM" OR "Building Information Modeling") } \\
\text { AND ("VR" OR "Virtual reality") AND ("Education" OR "student" } \\
\text { OR "design literacy") }\end{array}$ & $\begin{array}{l}\text { Limiters - Peer } \\
\text { Reviewed; Date } \\
\text { Published: 20200101- } \\
20201231 \\
\text { Narrow by Language: - } \\
\text { english } \\
\text { Search modes - } \\
\text { Boolean/Phrase }\end{array}$ & 169 \\
\hline $\begin{array}{l}\text { Engineering } \\
\text { Village }\end{array}$ & $\begin{array}{l}\text { (((((((("Design" OR "art* and craft*" OR "visual art*" OR } \\
\text { "architecture" OR "BIM" OR "Building Information Model*") } \\
\text { AND ("VR" OR "Virtual reality" OR "AR" OR "Augmented } \\
\text { reality") AND ("Education" OR "student" OR "design literacy")) } \\
\text { WN ALL)) AND ((\{ca\} OR \{ja\} OR \{cp\}) WN DT)) AND (\{english\} } \\
\text { WN LA)) AND ((2021 OR 2020) WN YR)) AND (\{oa\} WN ACT)) }\end{array}$ & & 63 \\
\hline
\end{tabular}

TABLE 3. Inclusion and exclusion criteria with their rationale used in the selection of articles (table inspired by Zlatanovic et al. [2017] and Fylkesnes [2018]).

\begin{tabular}{|c|c|c|}
\hline INCLUSION CRITERIA & EXCLUSION CRITERIA & RATIONALE \\
\hline $\begin{array}{l}\text { Peer-reviewed articles } \\
\text { Peer-reviewed conference } \\
\text { proceedings }\end{array}$ & Other formats & $\begin{array}{l}\text { Ensure scientific quality and decrease } \\
\text { the risk of inappropriate conclusions }\end{array}$ \\
\hline English language & Other languages & $\begin{array}{l}\text { Published for an international } \\
\text { audience in an accessible language }\end{array}$ \\
\hline Full text available & Full text not available & $\begin{array}{l}\text { Results of the studies are open for this } \\
\text { analysis as well as for inspection by } \\
\text { other researchers }\end{array}$ \\
\hline $\begin{array}{l}\text { Articles published between } 2015 \text { and } \\
\text { the search date }\end{array}$ & Articles published before 2015 & $\begin{array}{l}\text { Relevance to the current context of } \\
\text { the article }\end{array}$ \\
\hline $\begin{array}{l}\text { Articles concerning design or } \\
\text { architecture }\end{array}$ & $\begin{array}{l}\text { Other fields, such as medicine, } \\
\text { science, language, etc. }\end{array}$ & $\begin{array}{l}\text { Relevance to the article's guiding } \\
\text { questions }\end{array}$ \\
\hline $\begin{array}{l}\text { Articles where VR is used in a design } \\
\text { process as the main concern }\end{array}$ & $\begin{array}{l}\text { VR used in pure visualisation of } \\
\text { models, looking at simulations, visiting } \\
\text { virtual places, virtual classrooms, etc. } \\
\text { Testing of different VR equipment. } \\
\text { Articles that focus solely on learning } \\
\text { styles, pedagogical theories or } \\
\text { pedagogical approaches, etc. }\end{array}$ & $\begin{array}{l}\text { Relevance to the article's guiding } \\
\text { questions }\end{array}$ \\
\hline
\end{tabular}




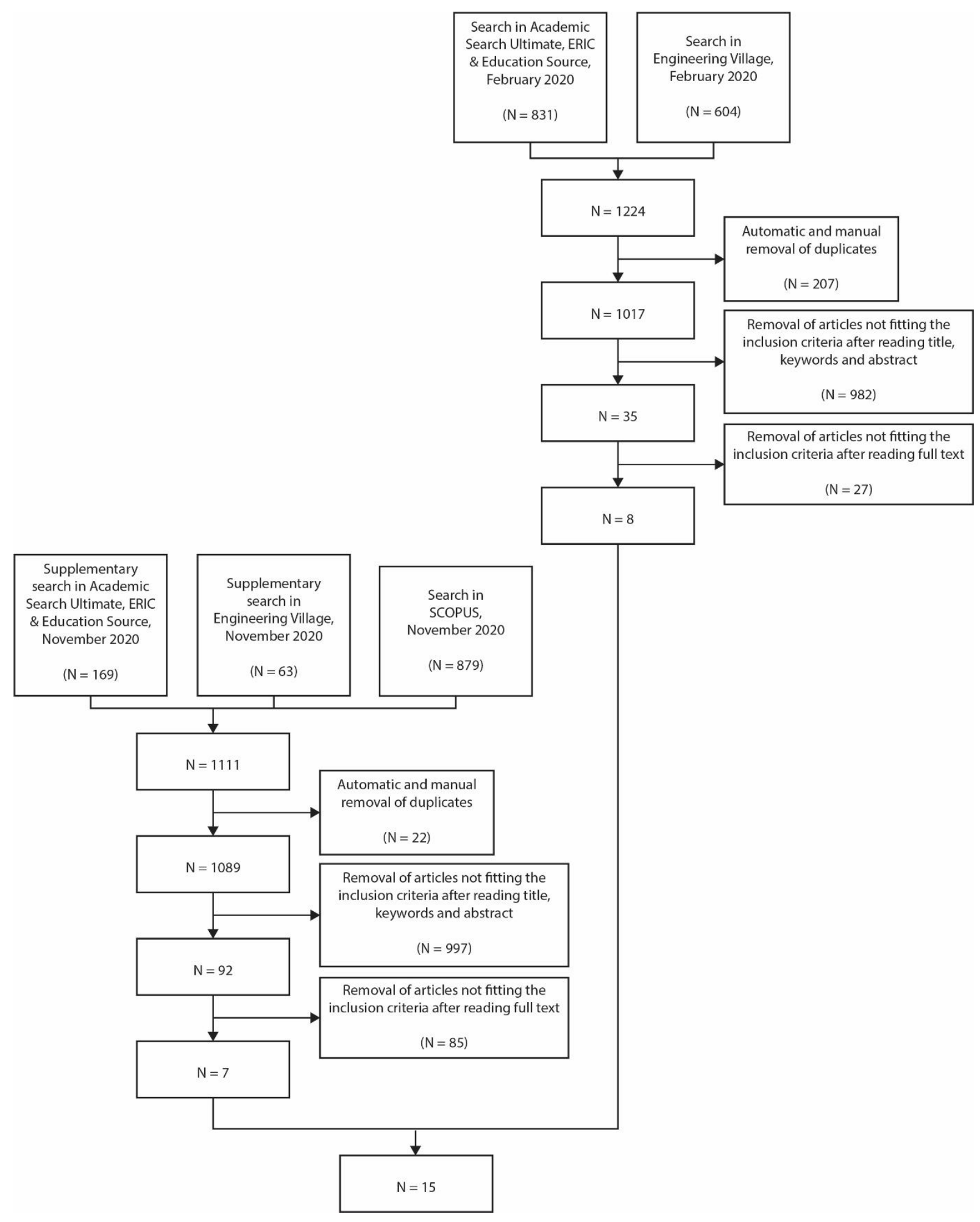

FIGURE 2. Flow chart visualising the process of selecting the articles.

Following this selection process, the number of included works was narrowed to 15 articles:

- Designing in caves: Using immersive visualisations in design practice. (Maftei \& Harty, 2015)

- Collaboration and dialogue in virtual reality. (Gyldendahl Jensen, 2017)

- Enabling participatory design of 3D virtual scenes on mobile devices. (Sun et al., 2017) 
- Augmented reality, virtual reality and their effect on learning style in the creative design process. (Chandrasekera \& Yoon, 2018)

- Development of spatial skills with virtual reality and augmented reality. (Gonzalez, 2018)

- Introducing virtual reality technologies to design education. (Häkkilä et al., 2018)

- A space design teaching model using virtual simulation technology. (Wu, 2018)

- Immersive virtual reality to enforce teaching in engineering education. (Halabi, 2019)

- The pedagogic value of learning design with virtual reality. (Nisha, 2019)

- Going immersive in a community of learners? Assessment of design processes in a multi-setting architecture studio. (Sopher et al., 2019)

- Enhancing learning and teaching for architectural engineering students using virtual building design and construction. (Zhang \& Chen, 2019)

- Virtual environments as medium for laypeople to communicate and collaborate in urban design. (Chowdhury \& Schnabel, 2020)

- $\quad$ "in VR, everything is possible!": Sketching and simulating spatially-aware interactive spaces in virtual reality. (Jetter et al., 2020)

- Using immersive virtual reality to support designing skills in vocational education. (Kim et al., 2020)

- Implementation and evaluation of a high-presence interior layout simulation system using mixed reality. (Lu \& Ishida, 2020)

The included articles make up only $0.71 \%$ of the total search results. The disciplines most prevalent among the excluded articles were medicine, or other health-related fields, and engineering, similar to the distribution described by Kavanagh et al. (2017, pp. 89-90), as well as computer science. As Freina and Ott (2015) reported in more detail, a substantial amount of research has been performed on the use of VR to simulate activities that otherwise would entail inconveniencies, risks or high costs, a trend that was also evident in these search results. As is apparent from the sheer number of excluded articles (2106) compared to included articles (15), little research has been conducted on the utilisation of VR technology in design processes. The low number of articles in the field of architecture and design is also evident in the review by Kavanagh et al. (2017), in which only 5 out of 99 papers belonged to architecture (3), interior design (1) or general art (1) (Kavanagh et al., 2017, p. 90). In the current review, the low number of included articles was partly due to choosing to include only those articles in which the main concern was the use of VR as part of the design process. In the last round, 91 articles, although fulfilling the other search criteria, were excluded because VR was not used in some sort of design process. Ultimately, the low number of included articles is one of the limitations of this review, as it decreases its generalisability and heightens the magnitude of the effect of potential flaws in each included article. Still, including all 91 of these articles would have meant including VR research with regard to the pure visualisation of models, simulations, visiting virtual places, virtual classrooms and art exhibitions, learning styles, pedagogical theories or pedagogical approaches, as well as the testing of different VR equipment. This would have generated broad data material ill-suited for answering the research questions.

Although limited in terms of sample size, the material included in this review had great geographical distribution. China is represented by three articles, and Great Britain by two, while the other ten articles are from Finland, Denmark, Austria, Switzerland, the USA, Mexico, Israel, Qatar, Japan and New Zealand. A data range spanning four continents despite the small sample size helped to secure valid results independent of context.

The findings from the included articles were analysed thematically in order to determine how each article described its use of VR and which benefits and challenges were reported from the studies. Given the small sample size, it was considered appropriate to present the use of VR in each study.

Further analysis identified categories of reported benefits and challenges, presented in their own sections below, in order to identify which themes were the most prevalent. 
The included studies employed mostly qualitative methods, except for Sun et al. (2017), Halabi (2019), Kim et al. (2020) and Lu and Ishida (2020), in which quantitative methods were used. Chandrasekera and Yoon (2018) employed 'a quantitative research design using analysis of subjective survey data' ( $p$. 61). The following methodologies were used in the included studies: Experiments (Chowdhury \& Schnabel, 2020; Kim et al., 2020; Lu \& Ishida, 2020; Sun et al., 2017; Zhang \& Chen, 2019), Case studies (Häkkilä et al., 2018; Maftei \& Harty, 2015; Sopher et al., 2019; Wu, 2018), Design-based research (Gyldendahl Jensen, 2017), Action research (Nisha, 2019), User study (Jetter et al., 2020) or Comparative study (Halabi, 2019). Data were gathered through surveys or questionnaires with some degree of selfassessment (Chowdhury \& Schnabel, 2020; Halabi, 2019; Häkkilä et al., 2018; Lu \& Ishida, 2020; Nisha, 2019; Sun et al., 2017; Wu, 2018; Zhang \& Chen, 2019), observation (Gonzalez, 2018; Gyldendahl Jensen, 2017; Jetter et al., 2020; Maftei \& Harty, 2015; Nisha, 2019; Sopher et al., 2019), interviews (Jetter et al., 2020; Nisha, 2019; Sopher et al., 2019), or audio-recordings from the experiment (Chowdhury \& Schnabel, 2020), as well as the collection of student projects (Nisha, 2019; Sopher et al., 2019), student grades (Halabi, 2019), participants' design assessments (Chowdhury \& Schnabel, 2020; Kim et al., 2020) or log data from the VR-interface (Kim et al., 2020; Sun et al., 2017). 13 of the 15 studies reported their number of participants. Apart from Nisha (2019), who had 85 participants, the numbers of participants were quite low, ranging from 10 to 35. Maftei and Harty (2015) and Gonzalez (2018) did not specify the number of participants. The employment of qualitative methods with small sample sizes means that the results are not easily amenable to generalisations but nonetheless constitute valuable contributions to a relatively young and small research field.

\section{PRESENTATION OF THE CHOSEN ARTICLES AND THEIR USE OF VR}

Laura Maftei and Chris Harty (2015) observed six sessions of collaborative design work in a full-scale 3D immersive environment - the CAVE - set up in a university lab. The observed project and design managers, architects, designers, modellers and visualisers were working on a large project: the design of a new hospital. Although the participants initially intended to use the CAVE technology for client presentations, they ultimately used it for making design decisions as well. With the help of a CAVE technician, the design group took virtual walks within a model of the planned building in order to evaluate its design and determine whether the design requirements were met.

Camilla Gyldendahl Jensen (2017) designed an immersive environment using a BIM model of a building to be renovated. The study was conducted at an event at the University College of Northern Denmark and involved 16 student volunteers from different educational programmes. The participants were divided into two groups to collaborate on the task, taking on different roles within the architecture, construction and engineering professions. Virtual walks inside the building were important to obtaining an understanding of the task, but the participants also held group meetings inside the virtual environment of the building. Although it was not clearly communicated, as the article's main focus was on collaboration, the groups' BIM team members might have been adjusting the BIM model accordingly between the meetings and virtual tours.

Xiaowen Sun, Yafang Wang, Gustave De Melo, Wei Gai, Yuliang Shi, Lu Zhao, Yulong Bian, Juan Liu, Chenglei Yang and Xiangxu Meng (2017) have developed a VR system for participatory design, involving a designer and a user. The designer works on a device with a 2D window on a screen, while the device simultaneously displays a corresponding 3D scene on a different screen. The user wears a mobile, immersive VR device that shows the 3D environment with the designer's changes in real time. The participants (user roles) are given the task to add furniture to an empty room in order to achieve an interior design to their liking. The users instruct the researcher (designer role) to place the furniture through verbal commands; meanwhile, they experience and evaluate the design as their commands are enacted in real time. Alternatively, the participants can use the 2D and 3D interface to design the interior by themselves, and then put on the VR glasses to check the simulation of their design.

Tilanka Chandrasekera and So-Yeon Yoon (2018) explored how learning preferences affect the use of AR and VR. In this study, which took place between December 2014 and April 2015, 30 design students used either a VR or AR system to solve a design problem in which an office space needed interior decorating. Both environments were non-immersive, viewed through a monitor, and looked 
and worked almost the same. What constituted the largest difference was that the VR system was controlled with a PC mouse, while the AR system was controlled through a set of physical fiducial markers.

Nora Argelia Aguilera Gonzalez (2018) discussed the use of VR and AR in a descriptive geometry course attended by students in their first and second semesters in architecture and industrial design. The technology is combined with traditional techniques, as the students start with sketching and drawing by hand before they make 3D models in SketchUp, which was the non-immersive VR employed in this study. The model is later converted to be viewed in AR in order to better check the model's scale and dimension, after which it is sent to a laser cutter to create a physical model.

Jonna Häkkilä, Ashley Colley, Jani Väyrynen and Antti-Jussi Yliharju (2018) studied the use of VR in three different cases, one of which is particularly relevant to the present article. In Case III, bachelor students studying industrial design used non-immersive VR or HMDs as part of their design process or in visualisation tasks. Their designs were later exhibited publicly through both immersive and nonimmersive VR, along with 3D-printed models and design posters.

Jialing Wu (2018) explored the use of a virtual teaching and training system in the architecture course 'Space Design'. Of interest to the present article is the training aspect of the course, in which the students worked with non-immersive VR to place different decorations in a virtual interior.

Cheng Zhang and Bing Chen (2018) designed an immersive VR environment consisting of three parts and tested it on 31 students from different domains in the built environment cluster, such as students of urban planning and architecture. In the context of the current article, the design aspect is especially interesting. Here, the students were given the task to conceive design ideas for a building, design the structure and interact directly with the environment. They were given an empty plot of land on which they could build their own building using rectangles, blocks and pre-made models in more complex shapes. The students could also leave tags with comments on each other's work, even though the environment only allowed for one user at a time.

Osama Halabi's (2019) study was set within the first year of an engineering study. The students worked in groups to design a wheelchair-accessible kitchen given specific constraints on room size, budget and materials. 3D models of the kitchen were made using the 3D-modeling software SketchUp, and a CAVE was used as the immersive virtual environment. The students could access the CAVE to test their prototypes, investigate the model and discover problems. In addition, two official sessions were arranged in the CAVE in which the groups presented their design to the rest of the class and to the instructor, who gave feedback on the design.

Bobby Nisha (2019) explored the pedagogic value of using HMD VR in two post-graduate, semester-long modules in Urban design, attended by students with a background in Urban design, planning, geography, civil engineering and GIS. In Module 1, the students designed a spatial composition of a typology of an urban landscape, incorporating elements such as paths, districts, edges, landmarks and nodes. The spatial compositions were later 3D-printed. In Module 2, students were asked to map the spatial evolution of cities and, based upon that, design an immersive, walk-through experience for VR.

Hadas Sopher, Dafna Fisher Gewirtzman and Yehuda E. Kalay (2019) analysed the design processes of architecture students who were using an IVE as a supplement to traditional studio work. In this study, the IVE was used for 'crits', whereby students presented their designs throughout the process to obtain feedback and input from teachers and peer students, as well as to study their own design in life-size virtual walkthroughs. Although the article was published in 2019, the data were gathered in the fall of 2013 and spring of 2016.

Shuva Chowdhury and Marc Aurel Schnabel (2020) have developed a collaborative VR tool for two or more users. Designer A wears an HMD, views the environment from a first-person point of view, and uses a controlling device to interact directly with $3 \mathrm{D}$ artefacts in the environment. Designer $\mathrm{B}$ views the environment through an 80-inch display and from a third-person point of view. This non-immersive set up means that Designer B can be more than one person. Designer(s) B interacts with the environment and the design by giving instructions to Designer $A$. With a target group of non-expert designers, Chowdhury and Schnabel set up an experiment involving volunteers with or without 3D- 
modelling skills. The participants were asked to design an urban space for an empty plot in a suburb, such as a playground, park, café, night market or food stall, using virtual 3D building blocks.

Hans-Christian Jetter, Roman Rädle, Tiare Feuchtner, Christoph Anthes, Judith Friedl and Clemens Nylandsted Klokmose (2020) have developed a VR tool for sketching and simulating interactive spaces containing tablets, large interactive screens, and futuristic devices, such as cylindrical or spherical touch screens. The VR tool is immersive, employing HMDs with finger- and hand-tracking, and allowing users to interact with virtual artefacts in the environment. In the user study, 12 interaction designers were invited to use the VR tool to freely design and sketch a multi-device interactive space.

Kevin Gonyop Kim, Catharine Oertel, Martin Dobricki, Jennifer K. Olsen, Alessia E. Coppi, Alberto Cattaneo and Pierre Dillenbourg (2020) investigated how immersive VR can support the designing skills of gardener apprentices compared to sketching with a pen or pencil on paper. The researchers developed a VR application, GardenVR, that has an HMD and controllers for both hands. The application has two modes: Design and Explore. The Design mode provides an exocentric view (top view of the garden) whereby participants can place trees, plants, etc. in the garden. The Explore mode provides an egocentric view where participants are inside their designed garden. In the 360-degree 3D environment, the garden can be explored via walkthroughs. The garden apprentices were given $2 \times 25$ minutes to design a garden room in their school garden. About half of the participants designed in the IVR in the first session and used traditional sketching in the second, while the other half used paper first and IVR second.

Yangzhicheng Lu and Tomoyuki Ishida (2020) developed a Mixed Reality interior layout system. Using a see-through HMD, users can scan the real interior space and then arrange virtual furniture within the environment created by the scan. An experiment was conducted with 22 university student volunteers to assess the presence, operability, functionality and effectiveness of the MR system.

\section{Summary of the articles' use of VR}

These articles demonstrate a variety of immersion levels. Immersive VR with HMDs was the most common level, used in six articles (Gyldendahl Jensen, 2017; Häkkilä et al., 2018; Jetter et al., 2020; Kim et al., 2020; Nisha, 2019; Zhang \& Chen, 2019). In addition, one article examined an MR system that employs see-through HMDs (Lu \& Ishida, 2020). Designing in non-immersive VR was reported in four of the articles (Chandrasekera \& Yoon, 2018; Gonzalez, 2018; Häkkilä et al., 2018; Wu, 2018). Two articles described systems in which immersive VR with HMDs and non-immersive VR were combined, allowing users to work together within different levels of immersion (Chowdhury \& Schnabel, 2020; Sun et al., 2017). Immersive Virtual Environments (IVE), such as the CAVE system, in which several users are placed in a dedicated room with screens or projections that cover three or four walls, the ceiling and/or the floor, were assessed in three articles (Halabi, 2019; Maftei \& Harty, 2015; Sopher et al., 2019).

In most of the included articles, VR systems were used as the main tool to work with complex tasks, such as designing buildings (Gyldendahl Jensen, 2017; Maftei \& Harty, 2015; Sopher et al., 2019; Zhang \& Chen, 2019), rooms (Halabi, 2019), urban spaces (Chowdhury \& Schnabel, 2020; Kim et al., 2020), products (Häkkilä et al., 2018) or other designs (Jetter et al., 2020; Nisha, 2019). On the other end of the scale, one study (Gonzalez, 2018) deployed VR as only one part of a process composed of traditional and technological techniques, physical and virtual materials. The last four studies (Chandrasekera \& Yoon, 2018; Lu \& Ishida, 2020; Sun et al., 2017; Wu, 2018) used VR in the main part of the design process, but the participants were given a simplified task of placing pre-made elements in a virtual space, as opposed to building a design from scratch and/or with higher degrees of creative freedom.

\section{BENEFITS FROM THE USE OF VR}

Through the analysis, four themes were identified as the most prevalent: a better understanding of size and proportions and of the design task; the facilitation of teamwork or peer participation; VR as motivating, fun, inspiring and an exciting experience; and the possibility of materialize one's ideas 
through digital prototypes. These themes are described below under their respective headings before additional benefits from the use of VR are mentioned.

\section{A better understanding of size and proportions and of the design task}

The benefit that stood out most clearly across the articles was better understanding. More specifically, VR experiences may contribute to a better understanding of size and proportions (Häkkilä et al., 2018; Jetter et al., 2020; Kim et al., 2020; Maftei \& Harty, 2015; Nisha, 2019; Sopher et al., 2019), as the designs can be viewed in full scale as opposed to viewing small physical models or sketches or drawings. Sopher et al. (2018) exemplified this benefit by discussing a pair of students who immediately reacted to their own design when viewing it in the IVE. The students claimed that their original design spaces were claustrophobic, later changing them to be more spacious. Jetter et al. (2020) described how some of the designers leveraged the fact that they were immersed in a full-scale virtual environment to experience sizes in relation to their own bodies, or jumping or standing on their toes to gauge whether an item could be reached.

After viewing their designs in VR, some participants also reported having a better understanding of complex issues concerning their design task (Gyldendahl Jensen, 2017; Sopher et al., 2019; Zhang \& Chen, 2019), as they could see more clearly which parts remained underdeveloped or needed to be changed, as well as how. Some participants commented that the VR environment enabled them to view their design in a way that would otherwise be impossible, such as from the perspective of being seated (Jetter et al., 2020; Maftei \& Harty, 2015). Design issues that would have been difficult or impossible to detect on a desktop presentation were also discovered in the IVE (Halabi, 2019; Maftei \& Harty, 2015).

Gonzales (2018) also argued that working with non-immersive VR gave the students a better understanding of volumetry as compared to working with projection drawings and axonometric drawings by hand.

\section{Facilitation of teamwork or peer participation}

The second clear benefit was the facilitation of teamwork or peer participation (Chowdhury \& Schnabel, 2020; Gyldendahl Jensen, 2017; Halabi, 2019; Maftei \& Harty, 2015; Nisha, 2019; Sopher et al., 2019) using VR. Out of these studies, three took place in an IVE (Halabi, 2019; Maftei \& Harty, 2015; Sopher et al., 2019), whereas one of the described systems (Chowdhury \& Schnabel, 2020) was specifically designed for collaboration between users. As the IVE is a dedicated room that can accommodate several people viewing the same projections, it is no surprise that it is an environment well suited for peer participation and group discussions on designs (Halabi, 2019; Maftei \& Harty, 2015; Sopher et al., 2019). Likewise, a system in which collaboration with others is a must in order to maximise its utility (Chowdhury \& Schnabel, 2020) can be expected to facilitate just that. HMDs, on the other hand, deliver sensory experiences to single individuals, meaning that others might be potentially excluded from these experiences. It is possible, through the use of avatars, to let users view each other in the virtual environment, but the HMDs are restricting them to view each other in the real world. The fact that two studies that employed HMDs also highlighted collaboration as one of the benefits shows that it is possible to design a VR task using HMDs that allow for collaboration, either working together on the creation of a VR experience (Nisha, 2019) or placing the participants both physically and virtually in the same environment to collaborate on a design task (Gyldendahl Jensen, 2017).

\section{Motivating, fun, inspiring and an exciting experience}

Several of the articles mentioned that the participants found the use of VR to be motivating in itself, fun, inspiring and an exciting experience (Halabi, 2019; Häkkilä et al., 2018; Jetter et al., 2020; Maftei \& Harty, 2015; Sun et al., 2017; Wu, 2018; Zhang \& Chen, 2019). In their review of literature on the use of VR in education, Kavanagh et al. (2017) identified a belief that the novelty of VR would be enough to motivate students. This factor also became apparent in the current review, with participants in the included studies for the most part experiencing VR in a design or study setting for the first time, although Häkkilä et al. (2018) mentioned that this effect may eventually wear off. As the novelty factor most likely diminishes with continual use (Kavanagh et al., 2017), it is interesting to note that this was not a focus 
in studies in which VR was used throughout a semester (Nisha, 2019; Sopher et al., 2019) or intensively for a short period (Gyldendahl Jensen, 2017). As a result, it is unclear from these studies whether the participants' motivation diminished along with the novelty factor, or if other benefits were more prevalent and interesting.

Reading the reports linked to motivation and excitement, it is also important to note the context from which the reports emerged, as, e.g. Wu (2018) opposed the use of VR in traditional learning processes, which were regarded as boring (p. 173). Maftei and Harty's (2015) observations of fun and excitement may also be seen in light of the collaborative aspect, as the design work in their study was considered 'fundamentally social and interactive' (p. 73), with descriptions of episodes as having 'a general tone of amusement, marked by loud laughing within the teams' members' ( $p .70)$.

\section{The possibility to materialize one's ideas through digital prototypes}

The last theme identified through the analysis was the possibility to materialize one's ideas through prototypes within virtual environments. Halabi (2019), who tested the application of VR in the first year of an engineering study, reported that some students expressed a sense of accomplishment upon viewing their designs in the CAVE. Although realised only in a virtual environment, the students were nonetheless satisfied that their work 'would not be just another abstract idea that would remain unimplemented on paper' (Halabi, 2019, p. 2997).

According to Chowdhury and Schnabel (2020), their VR design system enabled non-expert designers to produce meaningful 3D artefacts and to visualise pre-conceived design ideas. This could empower non-expert designers or laypeople to collaborate with designers in the early stages of urban design, as well as to participate in discussions regarding changes in their neighbourhoods.

In the user study conducted by Jetter et al. (2020), the ability to design and simulate interactions with futuristic devices, such as cylindrical or spherical screens, that would be impossible to make as physical prototypes, stood out as considerably important.

\section{Other benefits}

Other reported benefits of working with VR in the design process include allowing students to explore design theory in practice (Zhang \& Chen, 2019), as they can quickly construct a building and evaluate the results at full scale. This may, 'in return, improve their capability of applying, analyzing, synthesizing, evaluating or even creating new design principles' (Zhang \& Chen, 2019, p. 55). According to Sopher et al. (2019), the use of the IVE increased the number of converging design decisions in the students' processes, meaning that it complemented the traditional studio work, where more divergent design decisions are made, to enable students to engage in a more diverse and complete design process. The study by Wu (2018) examined an online course with VR functions, including both online teaching and the aforementioned VR training tasks, in which students decorated an interior. The students found the VR system to be efficient and to stimulate their learning process, as well as to enable them to combine theory and practice through the VR system's case scenario (Wu, 2018). According to Halabi (2019), working in groups with 3D modelling and using the CAVE improved the students' ability to demonstrate problem-solving skills. In the experiment conducted by Sun et al. (2017), the participants who designed solely within HMDs reported being more immersed in the design process.

\section{CHALLENGES FROM THE USE OF VR}

The thematic analysis also revealed several challenges involved in the use of VR. The most reported challenges were that VR systems limit the sketching or design process, that participants are unable to exploit the full potential of VR, and that VR systems generate diverse physiological and psychological effects. These themes are described below under their respective subheadings, after which additional challenges associated with the use of VR are mentioned. 


\section{VR systems limit the sketching or design process}

The most widely reported challenge of VR systems is that they impose limitations on the users' design processes, such as unsatisfactory sketching opportunities (Gyldendahl Jensen, 2017; Zhang \& Chen, 2019), or on the users' designs, as they do not offer the needed functionalities (Häkkilä et al., 2018; Jetter et al., 2020; Nisha, 2019; Zhang \& Chen, 2019). These are serious challenges, as they can affect the products that can be created and may lead users to choose different methods in their design process. Kim et al. (2020) compared the results from participants who completed a design task with pen or pencil on paper before moving on to working in VR to participants who started the design task in VR before using traditional sketching tools. They found that the sketching mode outperformed the VR mode on the creativity grade, as the sketch-work submissions earned better assessments than the VR submissions. Upon further analysis, they concluded that the students who started with sketching received the best overall assessment, which could be explained by increasing their creativity by starting with the sketching and then being able to focus more on proportions in the VR-interface, having an initial sketch on hand.

\section{Participants are unable to exploit the full potential of VR}

In the case study by Gyldendahl Jensen (2017), which entailed the exploration of a complex BIM model with HMDs, the participants exhibited signs of being unable to exploit the method's full potential, as they expended so much energy navigating and controlling the system that they had difficulty fully focusing on the design task. They also experienced difficulties getting started without a systematic approach (Gyldendahl Jensen, 2017). According to Kim et al. (2020), the increased cognitive load in the VR system could act as a barrier to the students' creativity, as the students might expend too much energy learning how to use the system.

The participants in the case study by Maftei and Harty (2015) were unfamiliar with the CAVE setting and thus spent considerable time orienting themselves with the technology. In the included vignettes, a CAVE technician was relied upon for navigating the environment, thereby missing some of the natural movement around the environment, as reported by, e.g. Jetter et al. (2020).

\section{Physiological and psychological challenges}

HMDs are also linked with diverse physiological challenges, such as dizziness (Nisha, 2019; Zhang \& Chen, 2019), or that the HMDs are heavy, thus restricting their use to shorter sessions (Nisha, 2019) and precluding their use among people with glasses, travel/motion sickness or epilepsy (Nisha, 2019). In a study by Jason Lucas (2018) - an article that did not meet all the inclusion criteria for the present review but is notable here - physiological discomfort was reported to be minimal, but psychological discomfort was of greater concern. Participants in this study reported being uncomfortable using the technology in front of other people, as doing so meant occluding their own vision.

\section{Other challenges}

Other reported challenges are that the number of HMDs limits the number of users (Häkkilä et al., 2018), or even that only one user at a time is allowed in the environment (Zhang \& Chen, 2019) due to limitations in the system. Setting up the HMD system is also time-consuming (Häkkilä et al., 2018) if it does not have a dedicated space. Additionally, depending on the rendering quality, simulated environments may portray varying degrees of realism (Maftei \& Harty, 2015). Lastly, Nisha (2018), along with other authors (Aguayo et al., 2017; Fowler, 2015; Kavanagh et al., 2017), have called for stronger pedagogic anchoring to ensure that excitement over the technology does not overshadow the focus on learning (Nisha, 2019).

\section{DISCUSSION ON THE DESCRIBED USE OF VR AND LEVELS OF IMMERSION}

The reviewed articles described the use of $V R$ at different levels of immersion. The clearest benefits of using the system, such as increased understanding of size and proportions and of the design task, the facilitation of teamwork or peer participation, and the motivating and fun experience it provides for 
users, were reported almost exclusively in those studies that employed immersive VR, with the use of either HMDs or IVEs. At the same time, immersive VR was used in all studies that reported challenges, such as unsatisfactory functionalities for sketching or designing, participants being unable to exploit the full potential of VR, and problems specific to HMDs, e.g. dizziness and heavy headsets. As this article reviewed a small number of studies, it is difficult to determine which benefits and challenges were related to differences in research design and which could be linked directly to the VR system being used.

What does stand out, however, is that only those studies that employed HMDs found the VR system to be limiting in terms of the participants' sketching or design process. Five of these six studies reported on that challenge specifically (Gyldendahl Jensen, 2017; Häkkilä et al., 2018; Jetter et al., 2020; Nisha, 2019; Zhang \& Chen, 2019). The sixth study that used HMDs (Kim et al., 2020) did not include data on user experiences but did find that the group that worked on paper before using the VR system earned higher scores on both their creativity and overall assessments. This suggests that HMD VR does not yet have the functions needed to work effectively as the sole tool in a design process.

The majority of the included studies, 12 in total, described some version of immersive VR, either HMDs (Gyldendahl Jensen, 2017; Häkkilä et al., 2018; Jetter et al., 2020; Kim et al., 2020; Nisha, 2019; Zhang \& Chen, 2019), MR with HMDs (Lu \& Ishida, 2020), IVEs (Halabi, 2019; Maftei \& Harty, 2015; Sopher et al., 2019) or a combination of immersive and non-immersive VR (Chowdhury \& Schnabel, 2020; Sun et al., 2017). The six studies that described non-immersive VR used desktop VR (Chandrasekera \& Yoon, 2018; Gonzalez, 2018; Häkkilä et al., 2018; Wu, 2018) or, again, some combination of immersive and non-immersive VR (Chowdhury \& Schnabel, 2020; Sun et al., 2017). The prevalence of studies using immersive VR was much higher than what this 2:1 relation can account for, as most of the themes were built solely on the results from studies employing immersive VR. This may be explained by the fact that in three of the studies using desktop VR (Chandrasekera \& Yoon, 2018; Gonzalez, 2018; Wu, 2018), it was used only as one part of a task or the participants were given a simplified task. Another explanation could be that the research questions were centred around a comparison of $V R$ and $A R$, or $V R$ and traditional techniques or teaching methods, rather than an exploration of the VR system in itself. Put another way, as Freina and Ott (2015) stated in their literature review of the advantages and potentials of using immersive VR in education, it is only with immersive VR that VR technology can reach its full potential. The question should be asked: Is a high immersion level and enhanced capacity to view a design at full scale necessary to fully understand size, proportions and other issues of the given design task?

\section{TRANSFERABILITY OF VR IN LOWER AND UPPER SECONDARY SCHOOL}

One of the challenges associated with HMDs is that they limit the number of users. Having only one or a few HMDs available is clearly insufficient as a teaching tool for a whole class. Setting up HMD systems for each session is also time-consuming. As previously mentioned, cardboard VR is a cheaper and simpler alternative, one that might even be distributed 1:1, granted that pupils can use private mobile phones. There are challenges to this as well, however, and it might not be possible to realistically apply everywhere - but compared to expensive, more advanced VR systems, cardboard HMDs stand out as a viable approach to implementing immersive VR in secondary school.

Immersive VR seems to lack the necessary functionalities for sketching and design; and without further development, it might not be optimal for the whole design process. When given a design task to model architectural solutions to renew a built environment, pupils may do the actual modelling in desktop 3D-modelling software. During the process, the 3D model can easily be converted for viewing in VR using the pupils' own mobile phones and cardboard HMDs. With immersive VR, the models can be studied at full scale to facilitate a better understanding of size and proportions and to, e.g. determine whether windows allow enough light into the building. The pupils could also obtain a sense of accomplishment from viewing their architectural designs at full scale, a feat clearly difficult to replicate with physical materials. Using HMDs for brief periods of time could also prevent some of the physiological challenges associated with VR, as this increases with the time spent in the environment (Lucas, 2018). As an alternative to a complex design task, pupils may also work on simpler projects 
related to architecture or product design. They could use such projects to explore design theory in practice without spending too much time and materials on making prototypes, and yet still have opportunities to view drafts in a 3D virtual environment.

Pupils may also use immersive VR as a preparation for a design task in architecture, such as using HMDs to explore pre-made models that show different layouts of building units with the same area. This way, they can get an idea of size and proportions, and they can determine which solutions are sufficiently spacious or are too narrow. Simpler models are thus preferred, as complex models may lead users to expend too much mental energy on navigating rather than on learning.

Trying VR can be motivating in itself, as well as a fun and exciting experience, as mentioned in articles on the subject (Bashabsheh et al., 2019; Gonzalez, 2018; Halabi, 2019; Häkkilä et al., 2018; Jetter et al., 2020; Maftei \& Harty, 2015; Sun et al., 2017; Wu, 2018; Zhang \& Chen, 2019), but the novelty factor of the technology would most likely diminish with continual use (Kavanagh et al., 2017). Some of the reviewed articles involved studies in which participants were working with VR technology for the first time, while other articles covered more long-term studies in which architecture or design students worked with VR during a whole semester. As previously mentioned, those studies that applied VR over a long term or on an intensive basis did not discuss the novelty factor, making it difficult to infer whether the participants' motivation diminished or whether the other reported benefits of using the technology were more interesting to discuss. To further explore this subject, case studies in which VR is used continually throughout a project would yield valuable results.

\section{CONCLUSION}

The articles included in this review described diverse uses of VR in design processes: both nonimmersive and immersive VR, VR as a main tool in complex tasks, VR as a part of a larger project, or VR in simplified tasks. The review's small sample size with large differences in research designs precludes its use in drawing conclusions about which methods work best.

The most prevalent reported benefit was that VR permits a better understanding of complex issues associated with design tasks, as well as enhanced understanding of size and dimensions. The role of VR in facilitating teamwork or peer participation, which surprisingly included two studies employing HMDs, was another reported benefit. Other benefits include the experience of VR as fun, inspiring and exciting, as well as the opportunity to use the technology to materialize one's ideas through digital prototypes. One study also found that the use of IVEs complemented traditional studio work, giving students a more diverse and complete design process. The most prevalent, as well as the most concerning, reported challenge was that VR systems imposed limitations on users' design processes, as these systems may not offer satisfactory functionalities for sketching and designing. Using complex systems, the users expended so much energy navigating and controlling the system that they had difficulty fully focusing on the design task. The review also revealed physiological challenges, such as dizziness, heavy HMDs or the prohibitive nature of HMDs for people who wear glasses, experience travel/motion sickness, or have epilepsy. The number of HMDs also limits the number of users, and the system may additionally only allow one user at a time. It is also time-consuming to set up the VR system. Simulated environments may also portray too low degrees of realism. Finally, pedagogic anchoring of VR systems has been strongly recommended.

Building upon these findings, alternatives for implementing VR in Art and crafts in lower secondary school, as well as in related subjects in upper secondary school, was discussed. Using cardboard VR glasses was suggested as an inexpensive alternative, permitting an increase in the number of simultaneous users. As immersive VR systems seem to lack functionalities for sketching and designing, it may be preferable to design first on desktop 3D-modelling software and then to check the model in HMDs throughout the remaining process. How this can be integrated in an optimal workflow is an issue that requires further study. Immersive VR may also be used in preparations for an architecture project, assisting in the exploration of pre-made models that demonstrate different layouts of building units with the same area. Although the findings suggest that using VR enhances understanding of size and proportions in architectural design, further research is needed to understand the technology's strengths and weaknesses in comparison to current methods. As frequently discussed, VR can be a motivating 
experience in itself, although its novel appeal may decline over time. It would therefore be interesting to evaluate this issue further through case studies in which VR is used continuously throughout longterm projects. 


\section{REFERENCES}

Aguayo, C., Cochrane, T., \& Narayan, V. (2017). Key themes in mobile learning: Prospects for learner-generated learning through AR and VR. Australasian Journal of Educational Technology, 33(6), 27-40. https://doi.org/10.14742/ajet.3671

Bashabsheh, A. K., Alzoubi, H. H., \& Ali, M. Z. (2019). The application of virtual reality technology in architectural pedagogy for building constructions. Alexandria Engineering Journal, 58(2), 713-723. https://doi.org/10.1016/j.aej.2019.06.002

Baus, O., \& Bouchard, S. (2014). Moving from virtual reality exposure-based therapy to augmented reality exposure-based therapy: A review. Frontiers in Human Neuroscience, 8(112). https://doi.org/10.3389/fnhum.2014.00112

Chandrasekera, T., \& Yoon, S.-Y. (2018). Augmented reality, virtual reality and their effect on learning style in the creative design process. Design and Technology Education: An International Journal, 23(1), 55-75. https://ojs.lboro.ac.uk/DATE/article/view/2267/2601

Chen, T., Zhao, M., Shi, Q., Yang, Z., Liu, H., Sun, L., Ouyang, J., \& Lee, C. (2018). Novel augmented reality interface using a self-powered triboelectric based virtual reality 3D-control sensor. Nano Energy, 51, 162-172. https://doi.org/10.1016/j.nanoen.2018.06.022

Chowdhury, S., \& Schnabel, M. A. (2020). Virtual environments as medium for laypeople to communicate and collaborate in urban design. Architectural Science Review, 63(5), 451-464. https://doi.org/10.1080/00038628.2020.1806031

Fowler, C. (2015). Virtual reality and learning: Where is the pedagogy? British Journal of Educational Technology, 46(2), 412-422. https://doi.org/10.1111/bjet.12135

Freina, L., \& Ott, M. (2015). A literature review on immersive virtual reality in education: State of the art and perspectives. Conference Proceedings of eLearning and Software for Education (eLSE), (1), 133-141. https://doi.org/10.12753/2066-026X-15-020

Fylkesnes, S. (2018). Whiteness in teacher education research discourses: A review of the use and meaning making of the term cultural diversity. Teaching and Teacher Education, 71, 24-33. https://doi.org/10.1016/j.tate.2017.12.005

Gonzalez, N. A. A. (2018). Development of spatial skills with virtual reality and augmented reality. International Journal on Interactive Design and Manufacturing, 12(1), 133-144. https://doi.org/10.1007/s12008-0170388-x

Greenhalgh, T., Thorne, S., \& Malterud, K. (2018). Time to challenge the spurious hierarchy of systematic over narrative reviews? European Journal of Clinical Investigation, 48(6), e12931. https://doi.org/10.1111/eci.12931

Gyldendahl Jensen, C. (2017). Collaboration and dialogue in virtual reality. Journal of Problem Based Learning in Higher Education, 5(1), 85-110. https://doi.org/10.5278/ojs.jpblhe.v0i0.1542

Halabi, O. (2019). Immersive virtual reality to enforce teaching in engineering education. Multimedia Tools and Applications, 79, 2987-3004. https://doi.org/10.1007/s11042-019-08214-8

Häkkilä, J., Colley, A., Väyrynen, J., \& Yliharju, A.-J. (2018). Introducing virtual reality technologies to design Education. Seminar. Net: Media, Technology \& Life-Long Learning, 14(1), 1-12. https://journals.oslomet.no/index.php/seminar/article/view/2584

Jetter, H.-C., Rädle, R., Feuchtner, T., Anthes, C., Friedl, J., \& Klokmose, C. N. (2020). "in VR, everything is possible!": Sketching and simulating spatially-aware interactive spaces in virtual reality. Proceedings of the $2020 \mathrm{CHI}$ Conference on Human Factors in Computing Systems, 1-16, Article 3376652. https://doi.org/10.1145/3313831.3376652

Juraschek, M., Büth, L., Posselt, G., \& Herrmann, C. (2018). Mixed reality in learning factories. Procedia Manufacturing, 23, 153-158. https://doi.org/10.1016/j.promfg.2018.04.009 
Kavanagh, S., Luxton-Reilly, A., Wuensche, B., \& Plimmer, B. (2017). A systematic review of virtual reality in education. Themes in Science and Technology Education, 10(2), 85-119.

https://www.learntechlib.org/p/182115/

Kim, K. G., Oertel, C., Dobricki, M., Olsen, J. K., Coppi, A. E., Cattaneo, A., \& Dillenbourg, P. (2020). Using immersive virtual reality to support designing skills in vocational education. British Journal of Educational Technology, 51(6), 2199-2213. https://doi.org/10.1111/bjet.13026

Lu, Y., \& Ishida, T. (2020). Implementation and evaluation of a high-presence interior layout simulation system using mixed reality. Journal of Internet Services and Information Security, 10(1), 50-63. https://doi.org/10.22667/JISIS.2020.02.29.050

Lucas, J. (2018). Immersive VR in the construction classroom to increase student understanding of sequence, assembly, and space of wood frame construction. Electronic Journal of Information Technology in Construction, 23, 179-194. https://www.itcon.org/2018/9

Ma, M., \& Zheng, H. (2011). Virtual reality and serious games in healthcare. In S. Brahnam \& L. C. Jain (Eds.), Advanced computational intelligence paradigms in healthcare 6. Virtual reality in psychotherapy, rehabilitation, and assessment. Studies in Computational Intelligence. Vol 337 (pp. 169-192). Springer Berlin Heidelberg. https://doi.org/10.1007/978-3-642-17824-5_9

Maas, M. J., \& Hughes, J. M. (2020). Virtual, augmented and mixed reality in K-12 education: a review of the literature. Technology, Pedagogy and Education, 29(2), 231-249. https://doi.org/10.1080/1475939X.2020.1737210

Maftei, L., \& Harty, C. (2015). Designing in caves: Using immersive visualisations in design practice. International Journal of Architectural Research: ArchNet-IJAR, 9(3), 53-75. https://doi.org/10.26687/archnetijar.v9i3.693

Merchant, Z., Goetz, E. T., Cifuentes, L., Keeney-Kennicutt, W., \& Davis, T. J. (2014). Effectiveness of virtual reality-based instruction on students' learning outcomes in K-12 and higher education: A meta-analysis. Computers \& Education, 70(12). https://doi.org/http://dx.doi.org/10.1016/j.compedu.2013.07.033

Milgram, P., \& Kishino, F. (1994). A taxonomy of mixed reality visual displays. IEICE Transactions on Information and Systems, 77(12), 1321-1329. https://cin.ufpe.br/ in1123/material/Milgram_IEICE_1994.pdf

Nisha, B. (2019). The pedagogic value of learning design with virtual reality. Educational Psychology, 39(10), 1233-1254. https://doi.org/10.1080/01443410.2019.1661356

Parveau, M., \& Adda, M. (2018). 3iVClass: A new classification method for virtual, augmented and mixed realities. Procedia Computer Science, 141, 263-270. https://doi.org/10.1016/j.procs.2018.10.180

Sanchez-Vives, M. V., \& Slater, M. (2005). From presence to consciousness through virtual reality. Nature Reviews Neuroscience, 6(4), 332-339. https://doi.org/10.1038/nrn1651

Slater, M., Lotto, B., Arnold, M. M., \& Sánchez-Vives, M. V. (2009). How we experience immersive virtual environments: The concept of presence and its measurement. Anuario de Psicología, 40(2), 193-210. http://hdl.handle.net/10261/59506

Sopher, H., Fisher Gewirtzman, D., \& Kalay, Y. E. (2019). Going immersive in a community of learners? Assessment of design processes in a multi-setting architecture studio. British Journal of Educational Technology, 50(5), 2109-2128. https://doi.org/10.1111/bjet.12857

Sun, X., Wang, Y., Melo, G. D., Gai, W., Shi, Y., Zhao, L., Bian, Y., Liu, J., Yang, C., \& Meng, X. (2017). Enabling participatory design of 3D virtual scenes on mobile devices. WWW'17 Companion: Proceedings of the 26th International Conference on World Wide Web Companion, 473-482. https://doi.org/10.1145/3041021.3054173

Tussyadiah, I. P., Jung, T. H., \& Tom Dieck, M. C. (2017). Embodiment of wearable augmented reality technology in tourism experiences. Journal of Travel Research, 57(5), 597-611. https://doi.org/10.1177/0047287517709090

Wu, J. (2018). A space design teaching model using virtual simulation technology. International Journal of Emerging Technologies in Learning, 13(6), 163-175. https://doi.org/10.3991/ijet.v13i06.8585 
Zahabi, M., \& Abdul Razak, A. M. (2020). Adaptive virtual reality-based training: A systematic literature review and framework. Virtual Reality, 24, 725-752. https://doi.org/10.1007/s10055-020-00434-w

Zhang, C., \& Chen, B. (2019). Enhancing learning and teaching for architectural engineering students using virtual building design and construction. Higher Education Studies, 9(2), 45-56. https://doi.org/10.5539/hes.v9n2p45

Zlatanovic, T., Havnes, A., \& Mausethagen, S. (2017). A research review of nurse teachers' competencies. Studies in Vocational and Professional Education, 10(2), 201-233. https://doi.org/10.1007/s12186-016-9169-0 